\title{
A Case of Malignancy-Associated Cryoglobulinemia
}

\author{
C. Kennedy \\ R. Doyle \\ N. Mayer ${ }^{b}$ \\ M. Clarkson ${ }^{\mathrm{a}}$ \\ Departments of ${ }^{\mathrm{a}}$ Nephrology and ${ }^{\mathrm{b}}$ Histopathology, Cork University Hospital, Cork, Ireland
}

\section{Key Words}

Malignancy-associated cryoglobulinemia $\cdot$ Hematological malignancy $\cdot$ Renal biopsy . Immunoglobulin

\begin{abstract}
We present a case of type 1 cryoglobulinemia with an underlying hematological malignancy. Unusually, the entire unifying diagnosis was made on the basis of the renal biopsy. Initially, serum cryoglobulin was not identified; repeat samples were positive. It is important to note that there is a significant false-negative rate with laboratory testing for cryoglobulin, due, at least in part, to the specific conditions required for collection and processing. With that in mind, in all cases with strong clinical or histological evidence of cryoglobulin but negative testing, carefully repeated samples should be sent.
\end{abstract}

\section{Introduction}

Cryoglobulins are either immunoglobulins or a mix of immunoglobulin and complement, which precipitate when cooled. They typically develop in the context of malignancy, chronic infection or connective tissue disease. Appropriate serum sampling and handling conditions are very specific. There is a significant false-negative rate when testing for cryoglobulin, largely due to suboptimal sample collection and processing.

\section{Case Report}

Clinical History and Initial Laboratory Data

A 75-year-old male was referred to the nephrology service for evaluation of hematuria, proteinuria and an elevated serum creatinine. His past medical history was notable for type 2 diabetes mellitus.

C. Kennedy

Department of Nephrology

Cork University Hospital

Cork (Ireland)

E-Mail kennedyclaire@gmail.com 
On the initial assessment, he appeared unwell. He was hypertensive with pedal edema and had dipstick hematuria and proteinuria. His urinary protein leak approximated $2 \mathrm{~g} /$ day. His initial biochemical laboratory findings are summarized in table 1.

His antinuclear antibody was positive with a homogenous pattern and he was hypocomplementemic; however, his anti-double-stranded DNA was negative. Serum protein electrophoresis demonstrated a monoclonal IgG kappa band.

\section{Kidney Biopsy}

A renal biopsy was performed, which demonstrated a diffuse endocapillary proliferative glomerulonephritis (fig. 1). Capillary wall kappa, IgG, IgM and C3 were noted on immunofluorescence (fig. 2) and there were scattered subendothelial electron-dense immune-type deposits on electron microscopy. There was a florid intimal arteritis involving the interlobular caliber arteries. Several arterioles contained eosinophilic material within the vessel wall consistent with cryoprecipitates.

Remarkably, coincidental sampling of perinephric fat demonstrated infiltration by a monomorphic population of CD20-positive B lymphocytes, which was suggestive of a lowgrade B cell lymphoma (fig. 3).

\section{Diagnosis}

The suspected diagnosis was type 1 cryoglobulinemia due to an underlying lymphoproliferative disorder. Computed tomography of the thorax, abdomen and pelvis revealed moderate hepatosplenomegaly and enlarged lymph nodes in the anterior mediastinum. However, an initial attempt did not confirm the presence of serum cryoglobulin. Due to the strong histological evidence and supportive clinical, radiological and laboratory findings, further sampling was performed and, this time, was positive. A subsequent bone marrow trephine biopsy was consistent with a diagnosis of low-grade follicular B cell lymphoma.

\section{Clinical Follow-Up}

The patient's initial clinical course was complicated by a nosocomial pneumonia and respiratory failure. Following stabilization, the patient underwent combination chemotherapy (rituximab, cyclophosphamide, vincristine and prednisolone).

Three months after initiation of therapy, the patient was normotensive and euvolemic, without constitutional symptoms. His follow-up laboratory findings can be seen in table 1 .

\section{Discussion}

Cryoglobulins are serum immunoglobulins that precipitate, usually with complement components, in temperatures less than $37^{\circ} \mathrm{C}$ and redissolve upon warming [1]. Multiorgan damage can ensue, mediated by hyperviscosity and/or immune-mediated vasculitis [1]. Clinical manifestations are therefore myriad with ischemic and/or vasculitic phenomena as well as generalized systemic upset [1].

Cryoglobulins are subdivided into three main categories on the basis of the clonality of the immunoglobulins in question [2]. Type 1 (simple) cryoglobulinemia involves a monoclonal immunoglobulin, usually seen in the context of a hematological malignancy such as chronic lymphocytic leukemia, multiple myeloma or Waldenström's macroglobulinemia. Type 2 cryoglobulinemia has mixed monoclonal and polyclonal elements, usually seen in the setting of chronic infection, most commonly chronic hepatitis C. In type 3 cryoglobulinemia, polyclonal immunoglobulin precipitates, often in the setting of connective tissue disease. 
The vast majority of cases have an identifiable underlying cause. As treatment and prognosis are based almost entirely on the underlying cause, an intensive effort must be made to identify it [3]. Testing for cryoglobulin is perceived as awkward and difficult due to sampling prerequisites. There is also significant interlaboratory and intralaboratory variation in storage and testing [4]. As a result, cryoglobulin testing is probably underutilized in general.

The main sampling criterion is that the blood sample is taken in a pre-warmed collection tube and kept at $37^{\circ} \mathrm{C}$ on the way to and in the laboratory to avoid premature precipitation of the immunoglobulin and, therefore, a false-negative laboratory test [5].

In the laboratory, warm centrifugation occurs and the serum is cooled and observed at $4^{\circ} \mathrm{C}$ for cryoprecipitate formation, which can take several days in some cases. The cryoprecipitate is washed and re-warmed to $37^{\circ} \mathrm{C}$ where it dissolves again and is subjected to a variety of immunoanalyses to characterize the immunoglobulin and the complement components [5].

As in the case above, a significant false-negative rate exists when testing for serum cryoglobulin, largely due to handling and storage errors. With that in mind, if there is strong clinical suspicion for cryoglobulinemia but a cryoglobulin is not detected, careful repeat sampling and direct liaison with the laboratory are crucial.

\section{References}

1 Ramos-Casals M, Stone JH, Cid MC, et al: The cryoglobulinaemias. Lancet 2012;379:348-360.

2 Takada S, Shimizu T, Hadano Y, et al: Cryoglobulinemia (review). Mol Med Rep 2012;6:3-8.

3 Terrier B, Cacoub P: Cryoglobulinemia vasculitis: an update. Curr Opin Rheumatol 2013;25:10-18.

-4 Sargur R, White P, Egner W: Cryoglobulin evaluation: best practice? Ann Clin Biochem 2010;47:8-16.

5 Motyckova G, Murali M: Laboratory testing for cryoglobulins. Am J Hematol 2011;86:500-502.

Table 1. Laboratory results at initial presentation and three months post-treatment initiation

\begin{tabular}{lcc}
\hline & On admission & At 3-month follow-up \\
\hline Creatinine, mg/dl & 2.3 & 1.2 \\
Albumin, g/dl & 3.8 & 3.8 \\
Lactate dehydrogenase, U/l & 568 & 433 \\
C3, g/l & 0.98 & 1.31 \\
C4, g/l & $<0.08$ & $<0.08$ \\
IgG, g/l & 2.83 & 3.59 \\
IgA, g/l & 2.23 & 1.48 \\
IgM, g/l & 1.84 & 1.08 \\
Spot urinary protein: creatinine, $\mathrm{mg} / \mathrm{mmol}$ & 211 & 37 \\
\hline
\end{tabular}



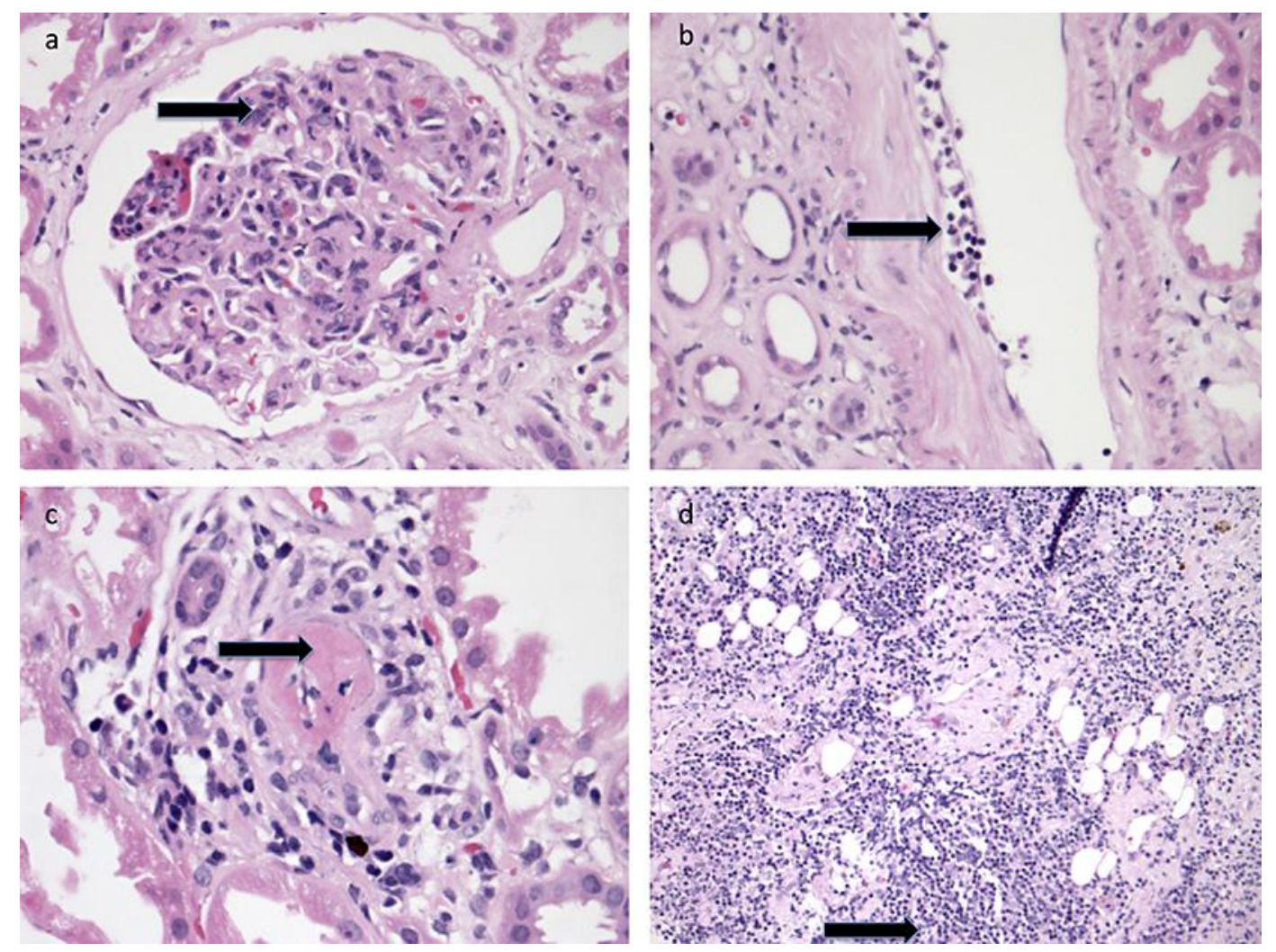

Fig. 1. a Hematoxylin and eosin staining (HE), high power. Florid endocapillary hypercellularity with prominent monocytes and granulocytes within capillary loops with minimal mesangial matrix expansion and variable capillary wall thickening, morphologically consistent with cryoglobulinemic glomerulonephritis. b HE, high power. Florid intimal arteritis involving the interlobular caliber artery. c HE, high power. Arteriole with inflammatory cell infiltration and eosinophilic material within the vessel wall consistent with cryoprecipitate. $\mathbf{d}$ HE, medium power. Monomorphic small lymphoid cells infiltrating the perinephric fat can be seen. 
Case Reports in

Nephrology and

Urology
Case Rep Nephrol Urol 2013;3:69-73

DOI: $10.1159 / 000353170$

Kennedy et al.: A Case of Malignancy-Associated Cryoglobulinemia
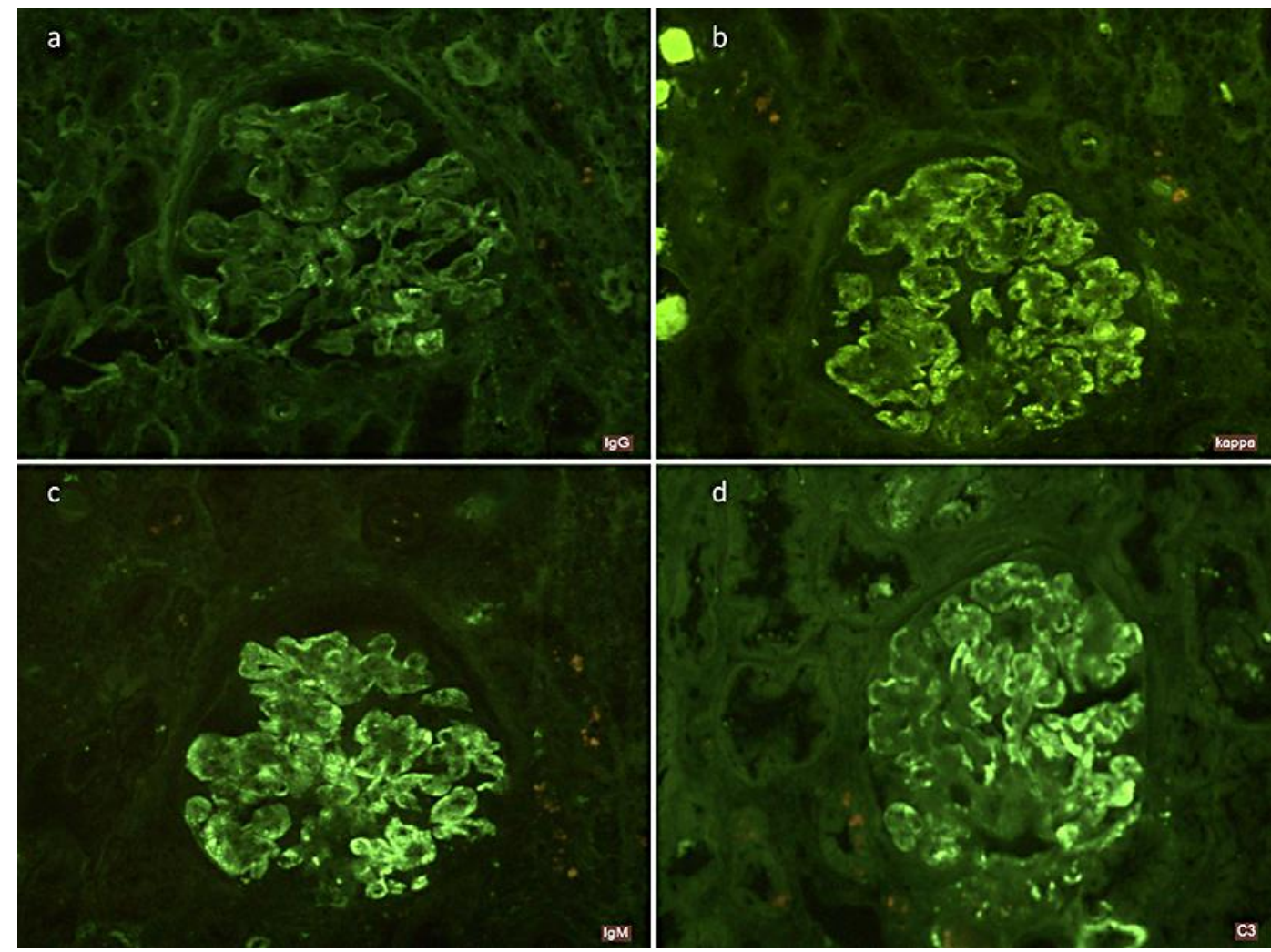

Fig. 2. Immunofluorescence demonstrated capillary wall positivity for kappa, IgM and C3 with negative IgG staining.

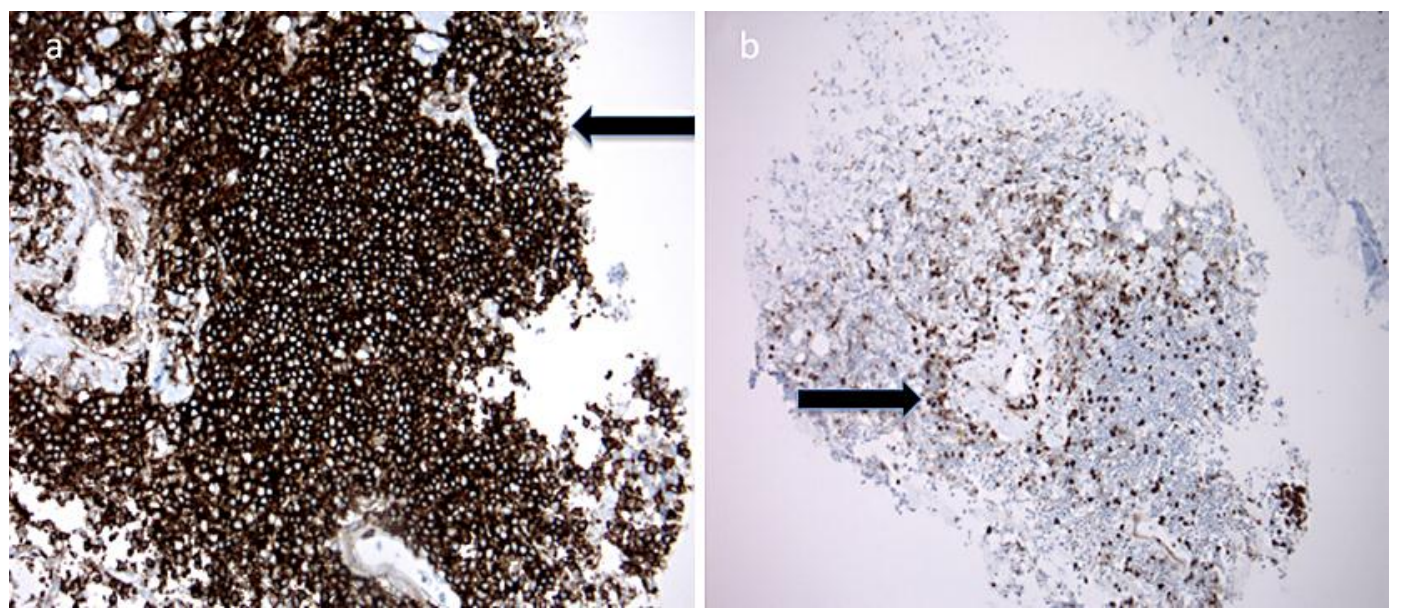

Fig. 3. a The lymphoid infiltrate showed strong diffuse CD20 staining. b CD3 staining highlighted only scattered reactive $\mathrm{T}$ lymphocytes. 